\title{
Complication of non-US guided procedure of aquafilling breast gel
}

\author{
Gozde Arslan ${ }^{1}$, Levent Celik ${ }^{1}$, Mehmet Mahir Atasoy ${ }^{1}$, Levent Celik ${ }^{2}$, Rahmi Cubuk ${ }^{1}$
}

${ }^{1}$ Maltepe University, Department of Radiology, ${ }^{2}$ Radiologica Imaging Center, Istanbul, Turkey

To the Editor,

Aquafilling gel is a less invasive breast augmentation method. It is also used as a dermal filler for face and buttocks. It requires only local anesthesia. The use of this gel is not FDA approved [1] and its use is prohibited in some countries [2]. It is injected into the breast tissue and most of the time it is applied in a random way without imaging guidance. We believe this procedure should be done under the guidance of ultrasonography (US) especially when used for the breast, as random applications may cause complications. The gel could leak into the neighbouring soft tissues (subpectoralis compartment, between the pectoralis muscle fibers, etc) and into the vascular compartment.

We present a 35 year old women who was referred to the radiology department with complaints of pain, redness, edema, and loss of normal shape and increased deformity of both breasts. She had a history of breast augmentation 8 months ago with aquafilling gel injection method. Based on her anamnesis, we learned that this procedure was applied by a clinician without the guiding of US. We performed US and magnetic resonance imaging (MRI). US showed bilateral edema of the breast and subglandular injected aquafilling material. The liquid material was heteregoneously and asymmetrically scattered through the fibroglandular tissue and also it had transpassed in between the pectoralis muscle fibers (fig 1a). There was also a hypoechoic solidified lesion on her left breast (figlb). We assumed this to be the gel which accumulated in a focal area. T2WS (T2 weighted sequence) of the MRI revealed the leakage of the aquafilling material into neighbouring tissues and into the subpectoral compartment.

Received 23.02.2017 Accepted 20.03.2017

Med Ultrason

2017, Vol. 19, No 2, 236-237, DOI: 10.11152/mu-1021

Corresponding author: Gozde Arslan

Basibuyuk mahallesi, Emek caddesi,

Narcity C1-26, Maltepe, Istanbul,Turkey

Phone: 905556880896

Email: gunesgozde@gmail.com

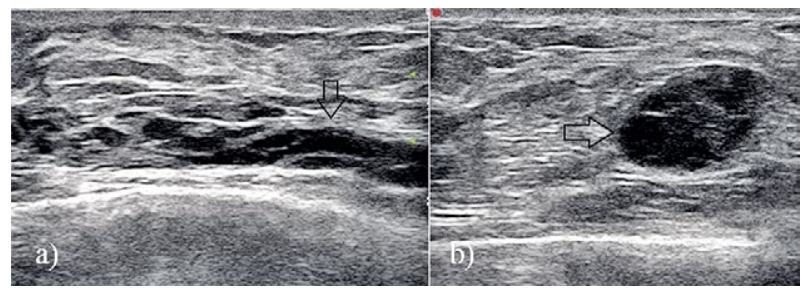

Fig 1. Sonography of the left breast: a) The liquid material was heteregoneously and asymmetrically scattered throughout the fibroglandular tissue and also it transpassed in between the pectoralis muscle fibers; b) Nodulary form of the gel material.

Our diagnosis was the leakage of the injected material and the fibroglandular tissue infection caused by it. The patient underwent surgery for removal of the injected material and antibiotics were started for the infection.

There are two breast augmentation methods. Silicone and saline implants are the most frequently used ones. They are placed into the subglandular or subpectoral compartment under general anesthesia. Despite being invasive, these methods are the most preferred and most reliable ones. There are some other, less known methods such as the aquafilling gel method which is performed in some countries. Aquafilling gel is a hydrophilic gel composed of saline (98\%) and polyamide (2\%). The gel is injected under the fibroglandular tissue through the skin by a needle - 100-200 ml of gel can be injected into each breast. This procedure is short and is performed under local anesthesia. However, it does require training and experience. As in our case, if performed in a random fashion, the material may be injected into incorrect locations and despite being less invasive, some local/ systemic complications may occur: fever, breast swelling, redness, tenderness /pain, asymmetry and deformity [3-5]. Many cases of breast deformity after aquafilling injections are irreversible and even require breast reconstruction. The gel also may form hypoechoic nodules in time and may be misdiagnosed as breast tumours. One of the most fearsome and unwanted complications is intravascular leakage and embolus of the material. Aquafilling gel procedure for breast augmentation should 
be performed by imaging and by experienced hands only. Patient satisfaction cannot be assured as asymmetries of the breasts may occur and and what is worse, many complications some of which are life threatening, may be caused. We also think that silicone implants are superior to aquafilling gel procedures based on our own experience.

\section{References}

1. U S Food and Drug Administration. Soft tissue fillers: Dermal fillers. Silver Spring, MD: U. S. Food and Drug Administration; 2015 [cited 2015 Jun 26].
2. Roh TS. Position Statement of Korean Academic Society of Aesthetic and Reconstructive Breast Surgery: Concerning the Use of Aquafilling ${ }^{\circledR}$ for Breast Augmentation. Arch Aesthetic Plast Surg 2016;22:45-46.

3. Peters W, Fornasier V. Complications from injectable materials used for breast augmentation. Can J Plast Surg 2009; 17:89-96.

4. Patlazhan G, Unukovych D, Pshenisnov K. Breast reconstruction and treatment algorithm for patients with complications after polyacrylamide gel injections: a 10-year experience. Aesthetic Plast Surg 2013;37:312-320.

5. Ono S, Ogawa R, Hyakusoku H. Complications after polyacrylamide hydrogel injection for soft-tissue augmentation. Plast Reconstr Surg 2010;126:1349-1357.

\title{
Ultrasound imaging for posterior knee pain: tibial nerve schwannoma not popliteus muscle strain
}

\author{
Ke-Vin Chang1, Wei-Ting Wu' ${ }^{1}$, Levent Özçakar ${ }^{2}$
}

\begin{abstract}
${ }^{1}$ Department of Physical Medicine and Rehabilitation, National Taiwan University Hospital, Bei-Hu Branch and National Taiwan University College of Medicine, Taipei, Taiwan; ${ }^{2}$ Department of Physical and Rehabilitation Medicine, Hacettepe University Medical School, Ankara, Turkey
\end{abstract}

\section{To the Editor,}

A 40-year-old male, a frequent jogger, complained of right posterior knee pain after a sprain six months ago. There was no trauma on his affected knee at that time. He described gradual onset of pain at the proximal deep leg, causing difficulty to stand up from a knee-flexed posture. He denied any numbness or weakness over the affected limb. He had been previously diagnosed with chronic popliteus muscle strain in sev-

Received 28.02.2017 Accepted 26.03.2017

Med Ultrason

2017, Vol. 19, No 2, 237-238, DOI: 10.11152/mu-1024

Corresponding author: Ke-Vin Chang, $\mathrm{MD}, \mathrm{PhD}$

Department of Physical Medicine and

Rehabilitation, National Taiwan University

Hospital, Bei-Hu Branch and National Taiwan

University College of Medicine

Taipei, Taiwan

E-mail: kvchang011@gmail.com; pattap@pchome.com.tw eral sport medicine centers though medical and physical therapies had shown little benefit. Accordingly, he was referred for an ultrasound (US) guided injection of dextrose solution. The pre-procedural physical examination revealed only tenderness over his proximal calf but no palpable mass at the painful region. During the US examination of his knee, only minimal effusion was noticed in the suprapatellar pouch. Distal to the popliteal crease, a round hypoechoic mass was identified between the gastrocnemius and popliteus muscles. Situated besides the popliteal artery and the tibial nerve, the mass appeared hypovascular. Magnetic resonance imaging was compatible with a neurogenic tumor (fig 1, Supplementary material on journal website - Video 1). His complaints were completely resolved after surgical resection and histopathology evaluation revealed a schwannoma.

Popliteus muscle originates from the lateral femoral condyle and courses medio-caudally to insert on the posterior aspect of the tibia cranial to the soleal line. Contrac- 


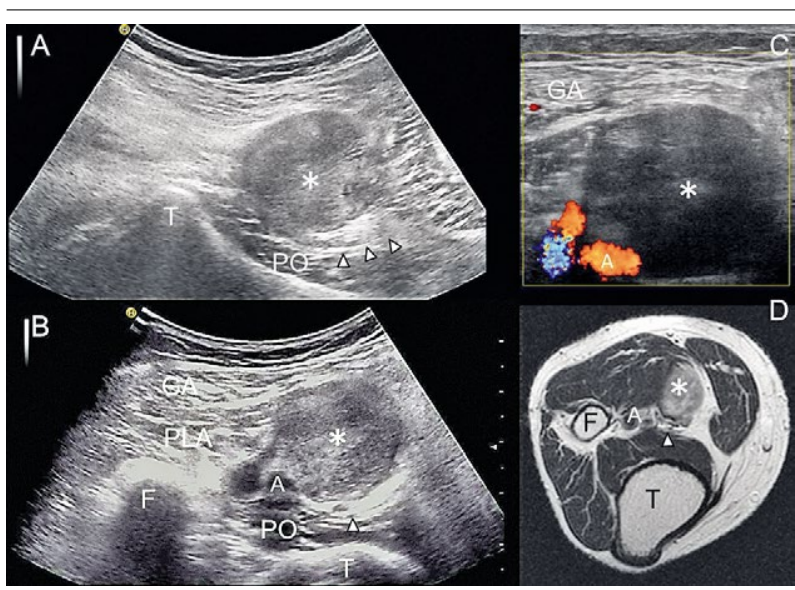

Fig 1. Ultrasound images of the schwannoma (asterisk) of the tibial nerve; long-axis (A), short axis (B) and color Doppler (C) images. Magnetic resonance imaging of the tumor in the horizontal plane (D). A: popliteal artery; white arrow: tibial nerve; PO: popliteus muscle; GA: gastrocnemius muscle; PLA: plantaris muscle; F: fibula; T: tibia.

tion of this muscle contributes to knee flexion and tibial internal rotation, unlocking the knee joint from an extended posture. The most common symptom of popliteus muscle strain is pain at the posterior knee, pain which can be reproduced by resistive knee flexion. US guidance is suggested for injection to this muscle, on top of which is the tibial nerve and a vascular network branched from the popliteal artery [1]. A curvilinear transducer is sometimes required for better visualization of the whole popliteus muscle because of its deep location. In patients with posterior knee pain, lesions of the posterior cruciate ligament and posterior horns of menisci should also be explored during the US examination. In our case, no associated lesions were recognized during initial US scanning, also excluding the possibility of acute popliteal muscle strain.

Tibial nerve schwannomas are benign tumors also uncommon causes of posterior knee pain. They grow slowly and rarely cause neurogenic complaints at the early stage [2]. The initial symptom is mostly pain caused by compression of the adjacent structures. Some later diagnosed patients may also present with radicular leg pain due to compressive neuropathy. The US diagnosis of a schwannoma is usually made by visualizing a well-bordered ovoid hypoechoic mass eccentrically accompanied by the nerve [3]. Their vascularity varies in individual cases and their connections with the nerves might sometimes be invisible [3]. In our case, the clinical presentation was quite similar to popliteus muscle strain. Since the mass was situated underneath the gastrocnemius muscle, it was not easy to sense its existence by palpation. Herein, it is noteworthy that familiarization with the local sonoanatomy serves as the key to correct diagnosis [4]. Since posterior knee is a less prevalent pain generator than its anterior, medial and lateral aspects, US should be used for scrutinizing uncommon pathologies and guiding interventions in cases of conservative treatment failure.

\section{References}

1. Chang KV, Hsiao MY, Hung CY, Ozcakar L. An Uncommon Cause of Posterior Knee Pain: Diagnosis and Injection for Popliteus Strain Using Ultrasonography. Pain Med 2016;17:795-796.

2. Ghaly RF. A posterior tibial nerve neurilemoma unrecognized for 10 years: case report. Neurosurgery 2001;48:668672.

3. Judd T, Jones T, Thornberry L. Schwannoma of the posterior tibial nerve: case study. J Am Podiatr Med Assoc 2014;104:539-543.

4. Hung CY, Hsiao MY, Ozcakar L, et al. Sonographic Tracking of the Lower Limb Peripheral Nerves: A Pictorial Essay and Video Demonstration. Am J Phys Med Rehabil 2016;95:698-708. 


\title{
Diagnosis of an actively bleeding rectus sheath hematoma by Contrast- Enhanced ultrasound
}

\author{
Tobias Kummer, Tyler M. Hartman, Jennifer L. White
}

Department of Emergency Medicine, Mayo Clinic, Rochester, Minnesota, USA

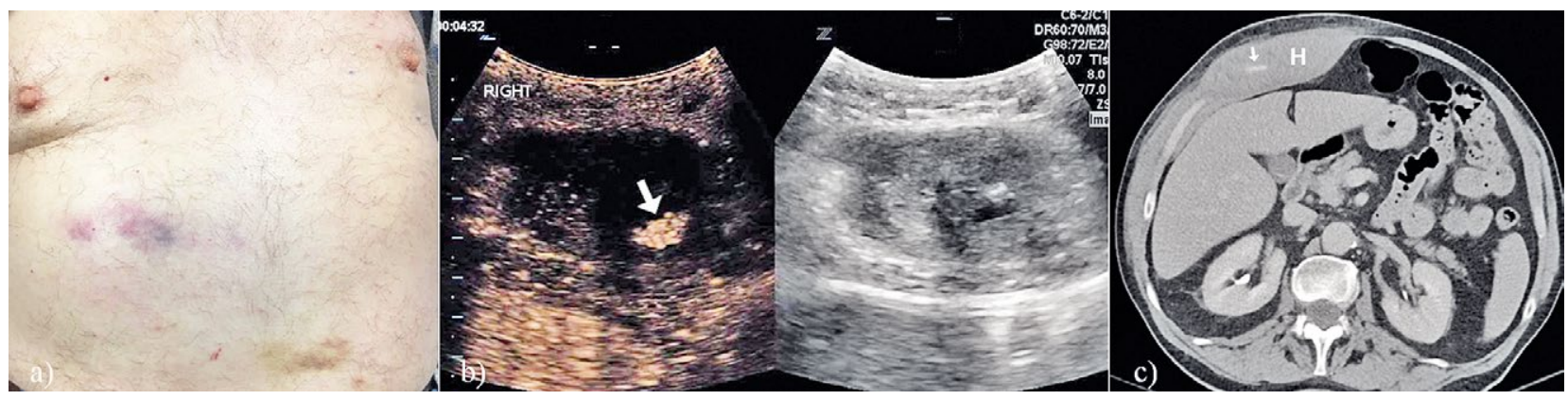

Fig 1. a) Abdominal ecchymosis and swelling in the right upper quadrant; b) Contrast-enhanced image shows contrast accumulation within the hematoma (arrow); c) Computed tomography. Hematoma $(\mathrm{H})$ is seen within the rectus sheath with contrast extravasation (arrow).

\section{To the Editor,}

An 81-year-old man presented to the emergency department with sudden swelling, ecchymosis, and pain in the right upper abdomen. He denied trauma or vomiting, but persistent, forceful coughing had kept him up at night. Anticoagulants included only $81 \mathrm{mg}$ of daily aspirin. On examination, the patient was uncomfortable and slightly pale with unremarkable vital parameters. The abdomen was notable for a large, swollen, and ecchymotic area of the right upper abdominal wall that was tender to palpation (fig 1a).

Laboratory results included a hemoglobin value of $10.9 \mathrm{~g} / \mathrm{dL}$. International normalized ratio and platelet count were within normal limits. Ultrasonography showed a large right rectus sheath hematoma. Lowmechanical index contrast-enhanced ultrasound (CEUS) with a bolus of $1.2 \mathrm{~mL}$ sulfur hexafluoride lipid-type A

Received 13.02.2017 Accepted 20.03.2017

Med Ultrason

2017, Vol. 19, No 2, 239-240, DOI: 10.11152/mu-1006

Corresponding author: Tobias Kummer, MD

Department of Emergency Medicine,

Mayo Clinic

200 First St SW, Rochester, MN 55905

E-mail: Kummer.Tobias@mayo.edu microspheres (Bracco Diagnostics, Inc) showed contrast accumulation within the hematoma, first detectable after approximately 90 seconds, consistent with ongoing, likely venous, bleeding (fig 1b). This finding was confirmed on computed tomography (CT), on which the hematoma measured 13.7/11.4/4.6 cm, and contrast extravasation was found in the delayed phase (fig 1c).

The patient was hospitalized and his aspirin therapy was held. He was discharged after his symptoms continued to improve, his hemoglobin level was stable, and the pain was well controlled.

Rectus sheath hematomas can be spontaneous or traumatic and are characterized by blood accumulation within the rectus sheath due to either tearing of the epigastric vessels or injury of the muscle itself, and their incidence appears to be rising because of increased use of anticoagulants among older patients [1]. Their management depends on the accurate diagnosis of any ongoing bleeding and identification of the type of injured vessel. Although most cases can be managed conservatively, patients with active bleeding should be monitored, and those with hemodynamic instability or ongoing blood loss might require embolization or surgical intervention [2].

The mainstay diagnostic imaging method is CT with intravenous contrast, which allows accurate assessment 
of the hematoma, including evaluation for contrast extravasation.

Whereas CT needs to be timed for the arterial or venous phase, modern ultrasound agents are stable for $5 \mathrm{~min}$ utes or more. Their utility has been shown by their high degree of diagnostic accuracy in endoleak detection after endovascular aneurysm repair [3] which is due to their strictly intravascular properties. The timing of contrast extravasation potentially allows differentiation of arterial from venous bleeding without the risks of contrast $\mathrm{CT}$ and with the advantage of being available at the point of care.

As shown by this case, CEUS can rapidly diagnose ongoing bleeding at the bedside. In addition, the time recording allowed determination of a venous bleed, which correlated with both the CT findings and the clinical picture. CEUS might be a promising alternative, particularly for patients in whom CT is either contraindicated or less desirable, and warrants further investigation.

\section{References}

1. Fitzgerald JE, Fitzgerald LA, Anderson FE, Acheson AG. The changing nature of rectus sheath haematoma: case series and literature review. Int J Surg 2009;7:150-154.

2. Salemis NS, Gourgiotis S, Karalis G. Diagnostic evaluation and management of patients with rectus sheath hematoma: a retrospective study. Int J Surg 2010;8:290-293.

3. Guo Q, Zhao J, Huang B, et al. A systematic review of ultrasound or magnetic resonance imaging compared with computed tomography for endoleak detection and aneurysm diameter measurement after endovascular aneurysm repair. J Endovasc Ther 2016;23:936-943. 